Volume 27 (2020) 43-60

DOI: $10.24330 /$ ieja.662949

\title{
FOR WHICH PUISEUX MONOIDS ARE THEIR MONOID RINGS OVER FIELDS AP?
}

\author{
Ryan Gipson and Hamid Kulosman \\ Received: 27 June 2018; Revised: 8 July 2019; Accepted: 9 July 2019 \\ Communicated by Abdullah Harmancı
}

\begin{abstract}
We characterize the Puiseux monoids $M$ for which the irreducible and the prime elements in the monoid ring $F[X ; M]$, where $F$ is a field, coincide. We present a diagram of implications between some types of Puiseux monoids, with a precise position of the monoids $M$ with this property.
\end{abstract}

Mathematics Subject Classification (2010): 13F15, 13A05, 20M25, 20M13, 13Gxx, 20M14

Keywords: Puiseux monoid, monoid domain $F[X ; M]$, AP domain, atomic domain, PC domain, gcd/lcm property, height $(0,0,0, \ldots)$, Prüfer monoid, integrally closed monoid

\section{Introduction}

If $M$ is a commutative monoid, written additively, and $F$ is a field, the monoid ring $F[X ; M]$ consists of the polynomial expressions (also called polynomials)

$$
f=a_{0} X^{\alpha_{0}}+a_{1} X^{\alpha_{1}}+\cdots+a_{n} X^{\alpha_{n}},
$$

where $n \geq 0, a_{i} \in F$, and $\alpha_{i} \in M(i=0,1, \ldots, n) . F[X ; M]$ is an integral domain if and only if $M$ is cancellative and torsion-free by [19, Theorem 8.1]. Since we will be dealing exclusively with cancellative torsion-free monoids, we will call monoid rings $F[X ; M]$, in that context, monoid domains. If $M=\mathbb{N}_{0}$, then $R=F[X ; M]=F[X]$, which is a PID and so, in particular, the notions of irreducible elements and prime elements coincide in $R$. The domains having this property are called AP domains. However, if $M=\langle 2,3\rangle=\{0,2,3,4, \ldots\}$ (the submonoid of $\mathbb{N}_{0}$ generated by the elements 2 and 3), then, for example, the elements $X^{2}$ and $X^{3}$ of $R$ are atoms that are not prime. If we do not restrict ourselves to submonoids of $\mathbb{N}_{0}$, but consider the submonoids of $\mathbb{Q}_{+}=\{q \in \mathbb{Q}: q \geq 0\}$ instead, then, for example, the monoid domain $F\left[X ; \mathbb{Q}_{+}\right]$is an AP domain (by a theorem of R. Daileda [12]), while in the monoid domain $F[X ; M]$ with $M=\left\langle\frac{1}{2}, \frac{1}{2^{2}}, \frac{1}{2^{3}}, \ldots ; \frac{1}{5}\right\rangle$ the element $X^{1 / 5}$ is an atom which is not prime (see [22]). So we naturally come to the following question: for which submonoids $M$ of $\mathbb{Q}_{+}$is the monoid domain $F[X ; M] A P$ ? The goal of this 
paper is to give an answer to this question. In addition to that, we will present an implication diagram between various properties of submonoids of $\mathbb{Q}_{+}$in which we will precisely position the monoids $M$ for which $F[X ; M]$ is AP.

Note that the additive submonoids of $\mathbb{Q}_{+}$have received a lot of attention by the researchers in the areas of commutative semigroup theory and factorization theory during the last few years (see, for example, [4,5,9,12,22,23,25,26,32]) and they even got a special name, Puiseux monoids, so we will be using that name from now on. Of course, Puiseux monoids were also used before; the paper [27] is one of the well-known instances. As we said, in this current paper we will be mainly dealing with the factorization properties of monoid domains $F[X ; M]$, where $F$ is a field and $M$ is a Puiseux monoid. Let us just mention that the factorization properties of general integral domains were thoroughly investigated in the papers by D. D. Anderson, D. F. Anderson, S. T. Chapman, J. Coykendall, W. W. Smith, M. Zafrullah, and many others (as stated in [14, page vii]).

\section{Notation and preliminaries}

We begin by recalling some definitions and facts. All the notions that we use but do not define in this paper, as well as the definitions and results for which we do not specify the source, can be found in the classical reference books [8] by P. M. Cohn, [18] and [19] by R. Gilmer, [32] by I. Kaplansky, and [35] by D. G. Northcott, as well as in our papers [5] and [22]. We also recommend the paper [1] in which the work of R. Gilmer is nicely presented, in particular his work on characterizing cancellative torsion-free monoids $M$ for which the monoid domain $F[X ; M]$ has the property $P$ for various properties $P$.

We use $\subseteq$ to denote inclusion and $\subset$ to denote strict inclusion. We denote

$\mathbb{N}=\{1,2, \ldots\}$ and $\mathbb{N}_{0}=\{0,1,2, \ldots\}$. An element $\frac{m}{n}$ of $\mathbb{Q}_{+}=\{q \in \mathbb{Q}: q \geq 0\}$ is said to be in reduced form if $\operatorname{gcd}(m, n)=1$.

All the monoids used in the paper are assumed to be commutative and written additively. Thus a monoid is a nonempty set $M$ with an associative and commutative operation $+: M \times M \rightarrow M$, possessing an identity element $0 \in M$. We say that a monoid $M$ is cancellative if for any elements $x, y, z \in M, x+y=x+z$ implies $y=z$. A monoid $M$ is torsion-free if for any $n \in \mathbb{N}$ and $x, y \in M, n x=n y$ implies $x=y$. If $M, M^{\prime}$ are Puiseux monoids, then every isomorphism from $M$ onto $M^{\prime}$ has the form $\mu_{\tau}(x)=\tau x$, where $\tau$ is a positive rational number (by [24, Proposition 3.2(1)]). Then $M^{\prime}=\tau M=\{\tau x: x \in M\}$. For a Puiseux monoid $M$, the difference group of $M$ is the subgroup $\operatorname{Diff}(M)=\{x-y: x, y \in M\}$ of $\mathbb{Q}$. 
A subset $I$ of a monoid $M$ is called an ideal of $M$ if $M+I=I$, i.e., if for every $a \in I, M+a \subseteq I$. (Here $S_{1}+S_{2}=\left\{x+y: x \in S_{1}, y \in S_{2}\right\}$ for any two subsets $S_{1}, S_{2}$ of $M$.) An ideal $I$ of $M$ is said to be principal if there is an element $a \in I$ such that $I=M+a$. We then write $I=(a)$. A submonoid of a monoid $M$ generated by a subset $A \subseteq M$ is denoted by $\langle A\rangle$ (while an ideal of $M$, generated by a subset $A \subseteq M$, is denoted by ( $A$ ), in order to avoid eventual confusions). We have: $\langle A\rangle=\left\{n_{1} a_{1}+\cdots+n_{t} a_{t}: t \geq 0, n_{i} \in \mathbb{N}_{0}, a_{i} \in A(i=1,2, \ldots, t)\right\}$. We assume $\langle\emptyset\rangle=\{0\}$. A monoid $M$ is said to be cyclic if it can be generated by one element. An element $a \in M$ is called an atom if $a$ is non-invertible and if $a=b+c$ $(b, c \in M)$ implies that at least one of the elements $b, c$ is invertible. We say that a monoid $M$ is atomic if every non-unit element of $M$ can be written as a sum of (finitely many) atoms. We say that a monoid $M$ is an $A C C P$ monoid if every increasing sequence

$$
\left(a_{1}\right) \subseteq\left(a_{2}\right) \subseteq\left(a_{3}\right) \subseteq \cdots
$$

of principal ideals of $M$ is stationary, meaning that $\left(a_{n_{0}}\right)=\left(a_{n_{0}+1}\right)=\left(a_{n_{0}+2}\right)=\ldots$ for some $n_{0}$. (Note that the notions of an atomic and an ACCP monoid are analogous to the notions of an atomic and an ACCP integral domain. The similarities and differences between the ideal theories of monoids and integral domains are studied, for example, in the classical references [3,29,31], as well as in the recent papers $[15,16,17]$.)

The notion of a Prüfer monoid was introduced in [21, p. 223-224] (see also [19, p. 166-167]). We include the possibility that $M=\{0\}$.

Definition 2.1. We say that a monoid $M$ is a Prüfer monoid it it is a union of an increasing sequence of cyclic submonoids.

We now give some observations and definitions in the context of Puiseux monoids. If $M$ is a Puiseux monoid and $A$ a generating set of $M$, note that:

(a) if $a$ is an atom of $M$, then $a \in A$;

(b) if $a \in A$ is such that $\langle A \backslash\{a\}\rangle \neq M$, then $a$ is an atom of $M$;

(c) if $a_{1}, \ldots, a_{n} \in A$ are not atoms of $M$, then $A^{\prime}=A \backslash\left\{a_{1}, \ldots, a_{n}\right\}$ is also a generating set of $M$.

Definition 2.2. We say that a Puiseux monoid $M$ is:

(a) difference-closed if for any $a, b \in M$ with $a \geq b$ we have $a-b \in M$;

(b) a half-group monoid if there is a subgroup $G$ of the additive group $\mathbb{Q}$ of rational numbers such that $M=G \cap \mathbb{Q}_{+}$; 
(c) integrally closed if for every $n \in \mathbb{N}$ and any $x, y \in M$ with $x \geq y, n(x-y) \in$ $M$ implies $x-y \in M$.

In this paper all rings are integral domains, i.e., commutative rings with identity in which $x y=0$ implies $x=0$ or $y=0$. A non-zero non-unit element $x$ of an integral domain $R$ is said to be irreducible (and called an atom) if $x=y z$ with $y, z \in R$ implies that $y$ or $z$ is a unit. A non-zero non-unit element $x$ of an integral doman $R$ is said to be prime if $x \mid y z$ with $y, z \in R$ implies $x \mid y$ or $x \mid z$. Every prime element is an atom, but not necessarily vice-versa. Two elements $x, y \in R$ are said to be associates if $x=u y$, where $u$ is a unit. We then write $x \sim y$.

We now give the definitions of some kinds of integral domains. The definitions of all other kinds that we use in the paper can be found in [19] and/or in the references given in the proof of Corollary 4.4 below.

Definition 2.3. An integral domain $R$ is said to be:

(a) an AP domain if every atom of it is prime;

(b) atomic if every non-zero non-unit element of it can be written as a (finite) product of atoms;

(c) a MIP domain if all maximal ideals of it are principal;

(d) a $P C$ domain if every proper two-generated ideal of it is contained in a proper principal ideal.

\section{Some properties of Puiseux monoids $M$ and the associated monoid} domains $F[X ; M]$

With respect to the AP-ness of the monoid domains $F[X ; M]$ for Puiseux monoids $M$ we have the next two propositions.

Proposition 3.1 ([10, Lemma 3.1]). Let $M$ be a Puiseux monoid, not isomorphic to $\mathbb{N}_{0}$. Then the irreducible elements of $F[X ; M]$ of the form $X^{a}, a \in M$, are precisely the $X^{a}$ with a an atom of $M$, and they are all non-prime.

Proposition 3.2 ([22, Theorem 4.2]). Let $M$ be a finitely generated Puiseux monoid. Then precisely one of the following situations occurs:

(a) $M=\{0\}$; then $F[X ; M]=F$, a field;

(b) $M=\langle a\rangle, a \neq 0$; then $F[X ; M] \cong F[X]$, a Euclidean domain;

(c) $M=\left\langle a_{1}, \ldots, a_{n}\right\rangle, n \geq 2$, all $a_{i}$ atoms of $M$; then $F[X ; M]$ is an atomic non-AP domain. 
To further study the AP-ness of the monoid domains $F[X ; M]$ for Puiseux monoids $M$ we introduced in [22] the following notion.

Definition 3.3 ([22]). We say that a Puiseux monoid $M$ satisfies the $\mathrm{gcd} / \mathrm{lcm}$ condition if for any $t \in \mathbb{N}$ and any elements $\frac{m_{1}}{n_{1}}, \frac{m_{2}}{n_{2}}, \ldots, \frac{m_{t}}{n_{t}} \in M$, written in reduced form, at least one of which is $\neq 0$, we have $\frac{\operatorname{gcd}\left(m_{1}, \ldots, m_{t}\right)}{\operatorname{lcm}\left(n_{1}, \ldots, n_{t}\right)} \in M$.

It is easy to see that an equivalent definition of the $\mathrm{gcd} / \mathrm{lcm}$ condition is that for any two elements $\frac{m_{1}}{n_{1}}, \frac{m_{2}}{n_{2}} \in M$, written in reduced form, at least one of which is $\neq 0$, we have $\frac{\operatorname{gcd}\left(m_{1}, m_{2}\right)}{\operatorname{lcm}\left(n_{1}, n_{2}\right)} \in M$.

The next proposition is a generalization of the theorem of Daileda, mentioned in Introduction. (The proof that we gave in [22] follows Daileda's proof of his theorem from [12].) The proposition is a step toward the main theorem of the paper, namely Theorem 4.3, and is in fact used in its proof.

Proposition 3.4 ([22, Theorem 5.4]). If a Puiseux monoid $M$ satisfies the gcd/lcm condition, then for any field $F$ the monoid domain $F[X ; M]$ is $A P$.

For a prime number $p$ the notion of the $p$-height $h_{p}(a)$ of an element $a$ of a torsion-free group $G$ is defined in [13, page 108 ] as the nonnegative integer $r$ such that $a \in p^{r} G \backslash p^{r+1} G$ if such an integer exists and as $\infty$ otherwise. The sequence $\left(h_{2}(a), h_{3}(a), h_{5}(a), \ldots\right)$ of $p$-heights of $a$ as $p$ goes through all prime numbers in the increasing order is called the height sequence of $a$. In our paper [5] we considered the elements of height $(0,0,0, \ldots)$, but, more generally, in the torsion-free monoids instead of groups.

Definition $3.5([5])$. We say that an element $a$ of a torsion-free monoid $M$ is of height $(0,0,0, \ldots)$ if for every prime number $p$ the equation $p x=a$ cannot be solved for an $x \in M$.

Example 3.6. Note that every atom of $M$ is an element of height $(0,0,0, \ldots)$. The converse does not hold. For example, in the monoid

$$
M=\left\langle\frac{1}{2}, \frac{1}{2^{2}}, \frac{1}{2^{3}} \ldots ; \frac{1}{3}, \frac{1}{3^{2}}, \frac{1}{3^{3}}, \ldots\right\rangle \subseteq \mathbb{Q}_{+}
$$

the element $\frac{5}{6}$ is of height $(0,0,0, \ldots)$, but is not an atom.

The next theorem is the main result of our paper [5]. It is not true for fields of positive characteristics (as the examples and related questions in [5] illustrate). We use this theorem in the proof of the main theorem of this paper (namely Theorem 
4.3) and that explains why we need in our main theorem the assumption that $F$ is of characteristic 0 .

Theorem 3.7 ([5, Theorem 4.1]). Let $M$ be a Puiseux monoid, $F$ a field of characteristic 0 , and $\pi$ an element of $M$ of height $(0,0,0, \ldots)$. Then the binomial $X^{\pi}-1$ is irreducible in $F[X ; M]$.

\section{When is $F[X ; M]$ an AP domain?}

In the next theorem we give several equivalent conditions for Puiseux monoids.

Theorem 4.1. Let $M$ be a Puiseux monoid. The following are equivalent:

(a) $M$ is a Prüfer monoid;

(b) $M$ is difference-closed;

(c) $M$ is a half-group monoid;

(c') $M=\operatorname{Diff}(M) \cap \mathbb{Q}_{+}$;

(d) $M$ satisfies the $\mathrm{gcd} / \mathrm{lcm}$ condition;

(e) $M \cong \mathbb{N}_{0}$ or $M$ has no elements of height $(0,0,0, \ldots)$;

(f) $M$ is integrally closed.

Proof. (a) $\Leftrightarrow\left(c^{\prime}\right) \Leftrightarrow(f)$ : follows from [19, Theorem 13.5].

$\left(c^{\prime}\right) \Leftrightarrow(c)$ : this is clear because of the minimality of the difference group $\operatorname{Diff}(M)$, which is precisely the Grothendieck group of $M$. (For the definition and properties of Grothendieck or universal group of $M$ see [28, Chapter II Section 2].)

(b) $\Leftrightarrow\left(c^{\prime}\right)$ : it suffices to observe that $M$ being difference closed is a restatement of $M=\operatorname{Diff}(M) \cap \mathbb{Q}_{+}$.

(b) $\Rightarrow$ (d): Let $\frac{m_{1}}{n_{1}}, \frac{m_{2}}{n_{2}}$ be two elements of $M$, written in reduced form, with $\frac{m_{1}}{n_{1}}<\frac{m_{2}}{n_{2}}$. Let $d=\operatorname{gcd}\left(m_{1}, m_{2}\right)$ and $e=\operatorname{gcd}\left(n_{1}, n_{2}\right)$, both positive. Then

$$
\begin{array}{rll}
m_{1}=d x_{1}, & m_{2}=d x_{2}, & \operatorname{gcd}\left(x_{1}, x_{2}\right)=1, \\
n_{1}=e y_{1}, & n_{2}=e y_{2}, & \operatorname{gcd}\left(y_{1}, y_{2}\right)=1, \quad \operatorname{lcm}\left(n_{1}, n_{2}\right)=e y_{1} y_{2} .
\end{array}
$$

Since $\operatorname{gcd}\left(x_{1} y_{2}, x_{2} y_{1}\right)=1$, there are $k, l \in \mathbb{N}_{0}$ such that

$$
d k x_{2} y_{1}-d l x_{1} y_{2}=d \text { or }-d
$$

We will assume that this difference is equal to $d$, the reasoning being similar if it is equal to $-d$. Since

$$
\frac{m_{1}}{n_{1}}=\frac{d x_{1}}{e y_{1}} \in M
$$


we have

$$
\frac{d l x_{1}}{e y_{1}} \in M
$$

Also

$$
\frac{m_{2}}{n_{2}}=\frac{d x_{2}}{e y_{2}} \in M
$$

hence

$$
\frac{d k x_{2}}{e y_{2}} \in M
$$

Hence, since $M$ is difference closed,

$$
\frac{d k x_{2}}{e y_{2}}-\frac{d l x_{1}}{e y_{1}} \in M
$$

i.e.,

$$
\frac{d k x_{2} y_{1}-d l x_{1} y_{2}}{e y_{1} y_{2}} \in M
$$

Hence by (1),

$$
\frac{d}{e y_{1} y_{2}} \in M
$$

i.e.,

$$
\frac{\operatorname{gcd}\left(m_{1}, m_{2}\right)}{\operatorname{lcm}\left(n_{1}, n_{2}\right)} \in M .
$$

Thus $M$ satisfies the gcd/lcm property.

$(\mathrm{d}) \Rightarrow(\mathrm{b})$ : Let $\frac{m_{1}}{n_{1}}, \frac{m_{2}}{n_{2}}$ be two elements of $M$, written in reduced form, with $\frac{m_{1}}{n_{1}}<\frac{m_{2}}{n_{2}}$. Let $d=\operatorname{gcd}\left(m_{1}, m_{2}\right)$ and $e=\operatorname{gcd}\left(n_{1}, n_{2}\right)$, both positive. Then

$$
\begin{array}{lll}
m_{1}=d x_{1}, & m_{2}=d x_{2}, & \operatorname{gcd}\left(x_{1}, x_{2}\right)=1, \\
n_{1}=e y_{1}, \quad n_{2}=e y_{2}, & \operatorname{gcd}\left(y_{1}, y_{2}\right)=1, \quad \operatorname{lcm}\left(n_{1}, n_{2}\right)=e y_{1} y_{2} .
\end{array}
$$

We have

$$
\frac{\operatorname{gcd}\left(m_{1}, m_{2}\right)}{\operatorname{lcm}\left(n_{1}, n_{2}\right)}=\frac{d}{e y_{1} y_{2}} \in M .
$$

Hence $M$ contains the element

$$
\frac{d}{e y_{1} y_{2}}=\frac{d\left(x_{2} y_{1}-x_{1} y_{2}\right)}{e y_{1} y_{2}}=\frac{d x_{2}}{e y_{2}}-\frac{d x_{1}}{e y_{1}}=\frac{m_{2}}{n_{2}}-\frac{m_{1}}{n_{1}} .
$$

Thus $M$ is difference closed.

$(\mathrm{e}) \Rightarrow(\mathrm{c})$ : Assume that $M$ has no elements of height $(0,0,0, \ldots)$ Let

$$
q_{1}, q_{2}, q_{3}, \ldots
$$


be the list of all the elements of $M$. Since $M$ has no elements of height $(0,0,0, \ldots)$, there are elements $\frac{q_{1}}{p_{1}}, \frac{q_{1}}{p_{1} p_{2}}, \frac{q_{1}}{p_{1} p_{2} p_{3}}, \ldots$ ( $p_{i}$ prime numbers) in $M$, so that the union $M_{1}$ of cyclic submonoids

$$
\left\langle q_{1}\right\rangle \subseteq\left\langle\frac{q_{1}}{p_{1}}\right\rangle \subseteq\left\langle\frac{q_{1}}{p_{1} p_{2}}\right\rangle \subseteq\left\langle\frac{q_{1}}{p_{1} p_{2} p_{3}}\right\rangle \subseteq \cdots
$$

is a (Prüfer, hence) half-group submonoid of $M$ containing $q_{1}$. Similarly, there is a half-group submonoid $M_{2}$ of $M$ containing $q_{2}$. Since the sum of two half-group Puiseux monoids is a half-group Puiseux monoid, the monoid $M_{1,2}=M_{1}+M_{2}$ is a half-group submonoid of $M$ containing $q_{1}$ and $q_{2}$, and $M_{1} \subseteq M_{1,2}$. Continuing in a similar way we construct an increasing sequence

$$
M_{1} \subseteq M_{1,2} \subseteq M_{1,2,3} \subseteq \cdots
$$

of half-group submonoids of $M$, such that $M_{1,2, \ldots, n}$ contains $q_{1}, q_{2}, \ldots, q_{n}(n=$ $1,2,3, \ldots$ ) Then the union

$$
M=M_{1} \cup M_{1,2} \cup M_{1,2,3} \cup \ldots
$$

is a half-group Puiseux monoid.

(a) $\Rightarrow$ (e): let $M=\cup_{n \in \mathbb{N}}\left\langle q_{n}\right\rangle$, where $\left\langle q_{1}\right\rangle \subseteq\left\langle q_{2}\right\rangle \subseteq \ldots$ Assume that $q \in M$ is of height $(0,0,0, \ldots)$, and take $N \in \mathbb{N}$ such that $q \in\left\langle q_{k}\right\rangle$ for $k \geq N$. Since $q$ has height $(0,0,0, \ldots)$, we find that $q_{k}=q$ for every $k \geq N$ and so $M=\left\langle q_{k}\right\rangle \cong \mathbb{N}_{0}$.

Corollary 4.2. Let $M, M^{\prime}$ be two isomorphic Puiseux monoids. Then $M$ satisfies any of the (equivalent) conditions from the previous theorem if and only if $M^{\prime}$ satisfies it.

We can now prove the main theorem of the paper. It characterizes the Puiseux monoids for which $F[X ; M]$ is an AP domain when $F$ is a field of characteristic 0 .

Theorem 4.3. Let $M$ be a Puiseux monoid and $F$ a field.

(i) Each of the conditions (a), (b), (c), (c'), (d), (e), (f) from Theorem 4.1 implies that $F[X ; M]$ is an AP domain.

(ii) If $F$ is of characteristic 0 , then each of the conditions from Theorem 4.1 is equivalent to $F[X ; M]$ being an AP domain.

Proof. (i) It is enough to show that one of the (equivalent) conditions (a)-(e) implies the condition $F[X ; M]$ AP. Hence the claim (i) follows Theorem 5.4 from our paper [22] (see the above Proposition 3.4) which states that if $M$ satisfies the gcd/lcm condition, then $F[X ; M]$ is AP. 
(ii) Because of (i) it is enough to show that the condition $F[X ; M]$ AP implies one of the (equivalent) conditions (a)-(e). We will show that it implies the condition (e). Let $F$ be a field of characteristic 0 . Assume $F[X ; M]$ is AP and $M$ is not isomorphic to $\mathbb{N}_{0}$. If $M=\{0\}$, the condition (e) holds. So we also assume that $M \neq\{0\}$. By Proposition 3.1 and Proposition 3.2, $M$ is infinitely generated and without atoms. Suppose to the contrary of the statement, i.e., that $M$ has an element of height $(0,0,0, \ldots)$, say $\pi$. Let $m, n \in \mathbb{N}$ and let $p$ be a prime number, such that $\operatorname{gcd}(m, n)=\operatorname{gcd}(p, n)=1$ and $n>1$. Let $q=\pi^{-1}(m / n+1 / p)$. The multiplication by $q$ is an isomorphism from $M$ to $q M$. The image $m / n+1 / p$ of $\pi$ is an element of height $(0,0,0, \ldots)$ in $q M$. Thus we can assume that $M$ has an element $\pi=(m / n+1 / p)$ of height $(0,0,0, \ldots)$, where $\operatorname{gcd}(m, n)=\operatorname{gcd}(n, p)=1$, $n>1$. We will show that then the element $X^{\pi}-1$ of $F[X ; M]$ is irreducible, but not prime. By Theorem 3.7, $X^{\pi}-1$ is irreducible in $F[X ; M]$. We now show that it is not prime. We have $\pi=\frac{m p+n}{p n}$. Hence

$$
X^{\pi}-1=\left(X^{\frac{m p+n}{p n}}-1\right) \mid\left(X^{\frac{m p+n}{p}}-1\right) .
$$

We have:

$$
\begin{aligned}
X^{\frac{m p+n}{p}}-1 & =\left(X^{\frac{1}{p}}\right)^{m p+n}-1 \\
& =\left(X^{\frac{1}{p}}-1\right)\left(X^{\frac{m p+n-1}{p}}+X^{\frac{m p+n-2}{p}}+\cdots+X^{\frac{1}{p}}+1\right) .
\end{aligned}
$$

Since $\frac{1}{p}<\pi$, we have $\left(X^{\pi}-1\right) \nmid\left(X^{\frac{1}{p}}-1\right)$. Suppose that

$$
\left(X^{\pi}-1\right) \mid\left(X^{\frac{m p+n-1}{p}}+X^{\frac{m p+n-2}{p}}+\cdots+X^{\frac{1}{p}}+1\right) .
$$

Then

$$
\begin{aligned}
X^{\frac{m p+n-1}{p}} & +X^{\frac{m p+n-2}{p}}+\cdots+X^{\frac{1}{p}}+1 \\
& =\left(X^{\frac{m p+n}{p n}}-1\right)\left(X^{\alpha_{1}}+g_{2} X^{\alpha_{2}}+\cdots+g_{k-1} X^{\alpha_{k-1}}-1\right),
\end{aligned}
$$

where $\alpha_{1}>\alpha_{2}>\cdots>\alpha_{k-1}>0$ and $g_{2}, \ldots, g_{k-1} \in F$. It follows that

$$
\alpha_{1}=\frac{(m p+n)(n-1)-n}{p n} .
$$

Note that

$$
\alpha_{1} \neq \frac{m p+n-i}{p} \text { for all } i=2,3, \ldots, m p+n-1,
$$

since, otherwise, we would get $(i-2) n=m p$, which is not possible since $\operatorname{gcd}(m, n)=$ 1 and $\operatorname{gcd}(p, n)=1$. Note also that

$$
\alpha_{1}<\frac{m p+n-2}{p}
$$


Hence the exponent $\frac{m p+n-2}{p}$ on the left-hand side of (3) has to be obtained from

$$
X^{\frac{m p+n}{p n}} \cdot\left(X^{\alpha_{1}}+g_{2} X^{\alpha_{2}}+\cdots+g_{k-1} X^{\alpha_{k-1}}-1\right),
$$

since it cannot be obtained from

$$
(-1) \cdot\left(X^{\alpha_{1}}+g_{2} X^{\alpha_{2}}+\cdots+g_{k-1} X^{\alpha_{k-1}}-1\right) .
$$

(These two expressions are parts of the right-hand side of (3).) But then we must have either

$$
\frac{m p+n}{p n}+\alpha_{2}=\frac{m p+n-2}{p}
$$

or, if

$$
\frac{m p+n}{p n}+\alpha_{i}=\frac{m p+n-2}{p} \text { for some } i \geq 3,
$$

then the terms with the exponents $\frac{m p+n}{p n}+\alpha_{2}, \ldots, \frac{m p+n}{p n}+\alpha_{i-1}$ would have to be cancelled on the right-hand side, so that we would have

$$
\begin{gathered}
\frac{m p+n}{p n}+\alpha_{2}=\alpha_{1}, \\
\frac{m p+n}{p n}+\alpha_{3}=\alpha_{2}, \\
\cdots \ldots \ldots \ldots \ldots \ldots \\
\frac{m p+n}{p n}+\alpha_{i-1}=\alpha_{i-2} .
\end{gathered}
$$

Hence

$$
\begin{aligned}
\alpha_{2} & =\frac{(m p+n)(n-2)-n}{p n}, \\
\alpha_{3} & =\frac{(m p+n)(n-3)-n}{p n}, \\
\ldots \ldots \ldots \ldots \ldots \ldots \ldots \cdots \cdots \cdots \cdots & \\
\alpha_{i-1} & =\frac{(m p+n)(n-i+1)-n}{p n},
\end{aligned}
$$

and from (5)

$$
\alpha_{i}=\frac{(m p+n)(n-i)-2 n}{p n} .
$$

Now since $\alpha_{i-1}>\alpha_{i}$, we would have

$$
\frac{(m p+n)(n-i+1)-n}{p n}>\frac{(m p+n)(n-1)-2 n}{p n},
$$

which gives

$$
n>(m p+n)(i-2) \text { for } i \geq 3,
$$


which is not true. Hence (4) holds. This gives

$$
\alpha_{2}=\frac{(m p+n)(n-1)-2 n}{p n} .
$$

Note that

$$
\alpha_{2} \neq \frac{m p+n-i}{p} \text { for all } i=3,4, \ldots, m p+n-1,
$$

since, otherwise, we would get $(i-3) n=m p$, which is not possible since $\operatorname{gcd}(m, n)=$ 1 and $\operatorname{gcd}(p, n)=1$. Note also that

$$
\alpha_{2}<\frac{m p+n-3}{p} .
$$

Hence the exponent $\frac{m p+n-3}{p}$ on the left-hand side of (3) has to be obtained from

$$
X^{\frac{m p+n}{p n}} \cdot\left(X^{\alpha_{1}}+g_{2} X^{\alpha_{2}}+\cdots+g_{k-1} X^{\alpha_{k-1}}-1\right)
$$

(we noticed before that it cannot be equal to $\alpha_{1}$ ).

Reasoning in the same way as before we conclude that

$$
\alpha_{3}=\frac{(m p+n)(n-1)-3 n}{p n} .
$$

By induction we get

$$
\alpha_{i}=\frac{(m p+n)(n-1)-i n}{p n}
$$

for $i=1,2,3, \ldots, r$, where $r$ is the largest integer for which

$$
\frac{m p+n}{p n}<\frac{m p+n-r}{p} \text {. }
$$

From (9) we get

$$
r<m p+n-1-\frac{m p}{n}
$$

Hence

$$
r \leq m p+n-2
$$

However, then

$$
\frac{m p+n-r}{p} \geq \frac{2}{p}
$$

so that not all of the exponents $\frac{m p+n-i}{p}$ from the left-hand side are obtained as $\frac{m p+n}{p n}+\alpha_{i}$. As before, $\alpha_{r}=\frac{(m p+n)(n-1)-r n}{p n}$ cannot be equal to any $\frac{m p+n-i}{p}, i=r+1, r+2, \ldots, m p+n-1$, otherwise we would get $n(i-r-1)=m p$, which is not possible since $\operatorname{gcd}(m, n)=1$ and $\operatorname{gcd}(p, n)=1$. Now note that

$$
\alpha_{r}<\frac{m p+n-(r+1)}{p} \text {. }
$$


It follows that the exponent $\frac{m p+n-(r+1)}{p}$ from the left-hand side cannot be obtained from

$$
X^{\frac{m p+n}{p n}} \cdot\left(X^{\alpha_{1}}+g_{2} X^{\alpha_{2}}+\cdots+g_{k-1} X^{\alpha_{k-1}}-1\right)
$$

since $r$ was the largest integer for which (9) holds, nor from

$$
(-1)\left(X^{\alpha_{1}}+g_{2} X^{\alpha_{2}}+\cdots+g_{k-1} X^{\alpha_{k-1}}-1\right)
$$

since (11) holds and no $\alpha_{i}$ with $i<r$ can be equal to any $\frac{m p+n-i}{p}, i=r+1, r+$ $2, \ldots, m p+n-1$. We got a contradiction, hence (2) does not hold. So $X^{\pi}-1$ is not prime.

The theorem is proved.

Corollary 4.4. The implications between some types of Puiseux monoids illustrated in Diagram 1 (see below) hold.

Proof. The implications in Diagram 1 are a direct consequence of Theorem 4.3, Theorem 4.1, [7, Theorem 2.3, Theorem 2.4, Theorem 2.8], [6, page 6], [19, Theorem 7.7, Corollary 12.11, Theorem 13.5, and page 169], [20, Theorem 8.4], [21, Theorem (iv)], [30, page 51], [32, page 114], [33, Proposition 3.1], and [36, Corollary 2.2, page 1896, page 1904].

\section{Concluding remarks}

(1) In our paper [22] we asked in Question 5.7 if for any Puiseux monoid $M$ without atoms $F[X ; M]$ is AP. We can now answer this question in the negative. Namely, the monoid

$$
M=\left\langle\frac{1}{2}, \frac{1}{2^{2}}, \frac{1}{2^{3}} \ldots ; \frac{1}{3}, \frac{1}{3^{2}}, \frac{1}{3^{3}}, \ldots\right\rangle \subseteq \mathbb{Q}_{+}
$$

that we considered in Example 3.6 does not have atoms, however by Theorem 4.3 $F[X ; M]$ is not AP for any field $F$ of characteristic 0 since $M$ contains elements of height $(0,0,0, \ldots)$, the element $\frac{5}{6}$, for example, being one of them. 


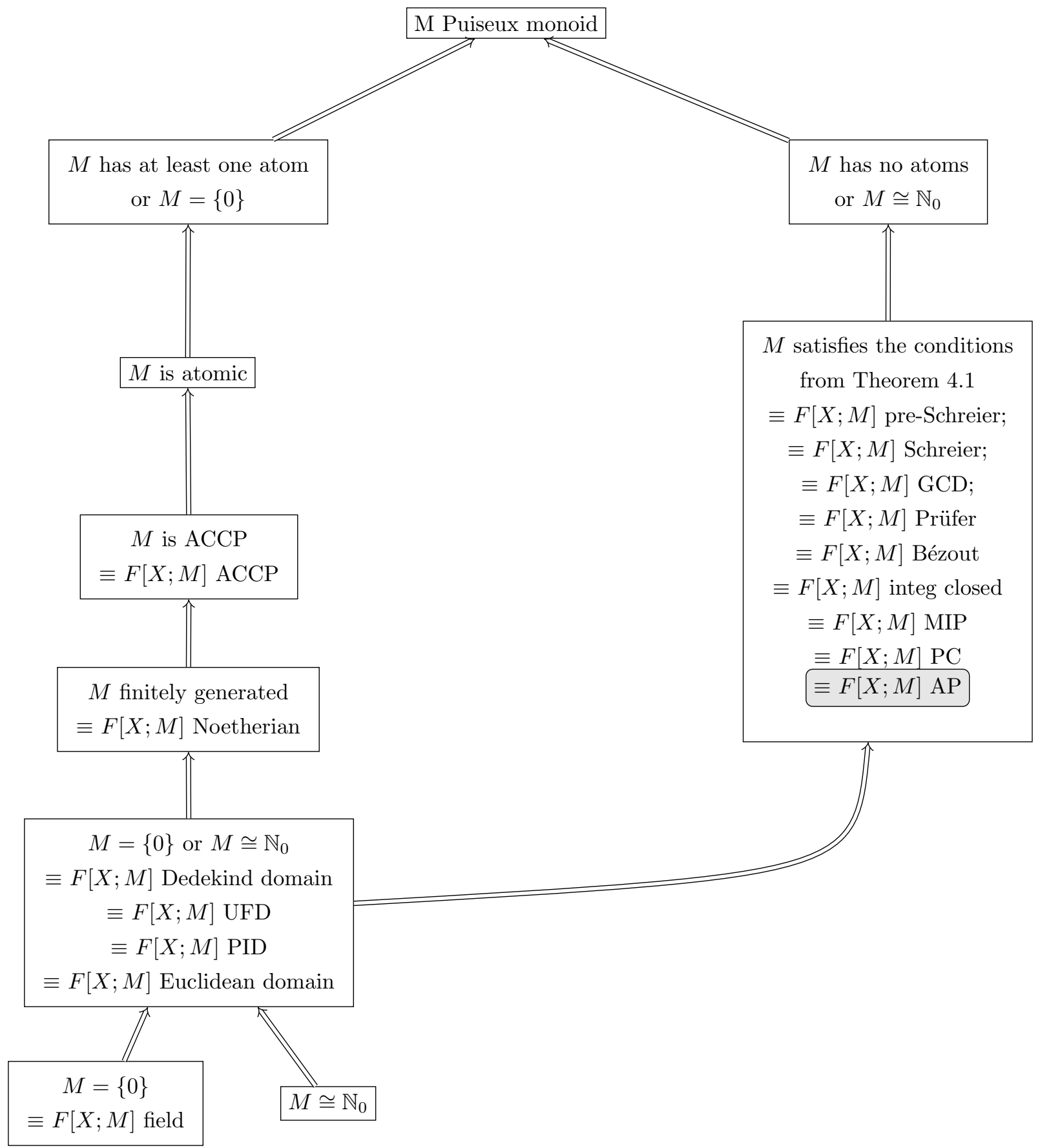

Diagram 1: Implications between some types of Puiseux monoids 
(2) In Question 5.7 from our paper [22] we also asked for a characterization of all Puiseux monoids $M$ such that $F[X ; M]$ is AP. The main theorem of this paper (namely, Theorem 4.3) answers this question for fields $F$ of characteristic 0 . In the proof of our main theorem we use Theorem 3.7 from our paper [5], which is not true for fields of positive characteristic. So we may naturally ask the following question: for a given characteristic $p>0$ characterize all Puiseux monoids $M$ such that $F[X ; M]$ is AP for any field $F$ of characteristic $p$. In particular, characterize all Puiseux monoids $M$ such that $F[X ; M]$ is AP for any field $F$ of positive characteristic.

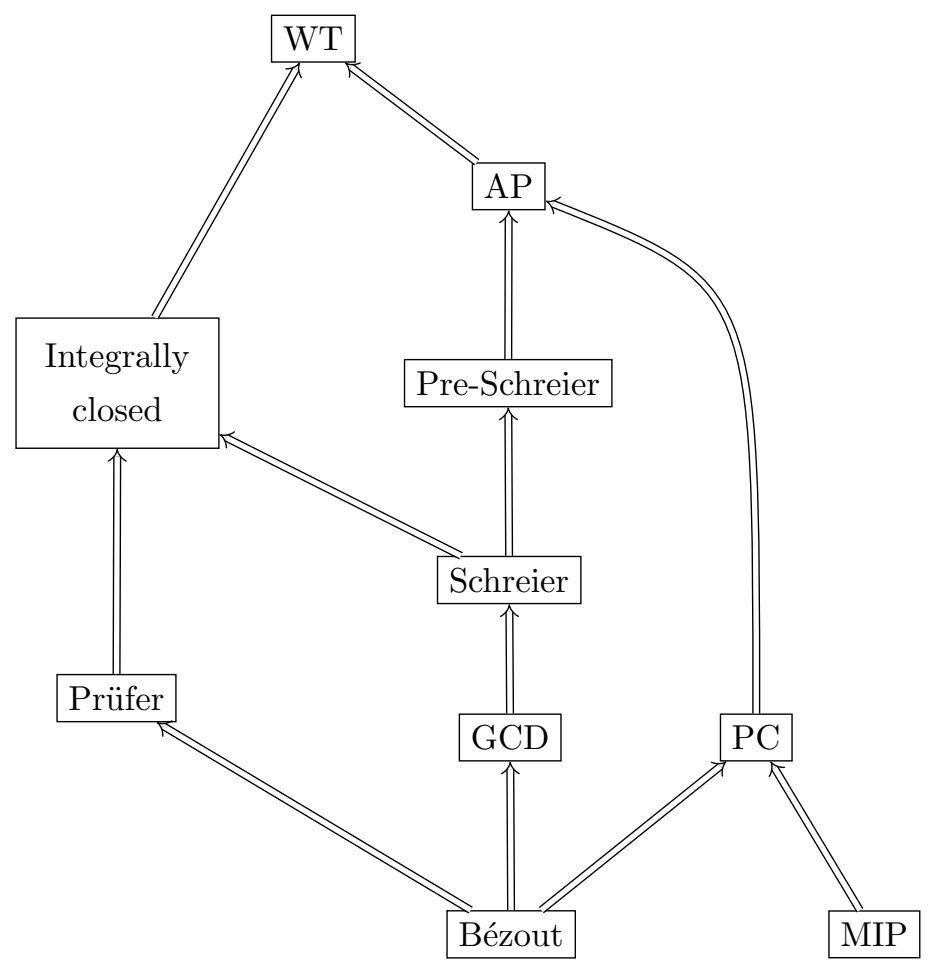

Diagram 2: Some equivalent types of domains in the $F[X ; M]$ context with $M$ a Puiseux monoid

$(3)^{1}$ Another question that we had in [22] was Question 2.11 in which we asked if for every atomic Puiseux monoid $M, F[X ; M]$ is atomic. (In other words, if $M$ atomic is equivalent with $F[X ; M]$ atomic, as the other direction was proved in our

${ }^{1}$ This question was recently answered in the negative by J. Coykendall and F. Gotti in [9]. 
[22, Proposition 2.10].) This question is a special case of the question of Gilmer ([19, page 189]) about determining the conditions under which the domain $D[X ; M]$ is atomic, where $D$ is a domain and $M$ is a cancellative torsion-free monoid. For the case of torsion-free abelian groups instead of monoids, the question was considered and some results obtained in [34, Section 8]. (The atomic structure of Puiseux monoids was recently investigated in several papers of F. Gotti, see for example [23].)

(4) The following is a natural question about Diagram 1: what would be the weakest type (WT) of integral domains such that all integrally closed domains and all AP domains are WT, and such that all the types of domains from Diagram 2 above are equivalent in the context of the monoid domains $F[X ; M]$, where $M$ is a Puiseux monoid?

(5) In [11] the notion of U-UFD is defined. It was shown that every AP domain is U-UFD and an example is constructed of an U-UFD which is not AP. A question one can ask is if the notions of AP and U-UFD are distinct in the context of the monoid domains $F[X ; M]$ of Puiseux monoids $M$, and if they are, to characterize the Puiseux monoids $M$ for which $F[X ; M]$ is a U-UFD. One could then use this characterization to obtain other examples of U-UFD's that are not AP.

(6) Another question one can consider is to characterize the Puiseux monoids $M$ for which the monoid domains $F[X ; M]$ are IDF, HFD, FFD, BFD (these domains and relations between them are analyzed in detail in [2]).

Acknowledgement. The authors would like to thank the anonymous referee for a very detailed review, many excellent remarks, comments, suggestions, shorter proofs. All that has significantly improved this paper.

The authors are also thankful to Steve Seif for his comments on an earlier draft of the paper and, in particular, for mentioning that the essential generators of a monoid $M$ (the notion that we introduced in [22]) are, in the context of Puiseux monoids, precisely the atoms of $M$.

\section{References}

[1] D. F. Anderson, Robert Gilmer's work on semigroup rings, in "Multiplicative Ideal Theory In Commutative Algebra, A tribute to the work of Robert Gilmer" (J. Brewer et al. (Eds)), Springer Science+Business media, LLC, (2006), 21-37. 
[2] D. D. Anderson, D. F. Anderson and M. Zafrullah, Factorization in integral domains, J. Pure Appl. Algebra, 69(1) (1990), 1-19.

[3] K. E. Aubert, Theory of $x$-ideals, Acta Math., 107 (1962), 1-52.

[4] S. T. Chapman, F. Gotti and M. Gotti, Factorization invariants of Puiseux monoids generated by geometric sequences, to appear in Comm. Algebra, DOI: 10.1080/00927872.2019.1646269; also arXiv1904.00219.

[5] K. Christensen, R. Gipson and H. Kulosman, Irreducibility of certain binomials in semigroup rings for nonnegative rational monoids, Int. Electron. J. Algebra, 24 (2018), 50-61.

[6] K. Christensen, R. Gipson and H. Kulosman, A new characterization of principal ideal domains, arXiv 1805.10374v1 [math.AC].

[7] P. M. Cohn, Bezout rings and their subrings, Proc. Cambridge Philos. Soc., 64 (1968), 251-264.

[8] P. M. Cohn, Algebra, Vol. I, Second Edition, John Wiley \& Sons Sons, Ltd., Chichester, 1982.

[9] J. Coykendall and F. Gotti, On the atomicity of monoid algebras, J. Algebra, 539 (2019), 138-151.

[10] J. Coykendall and B. Johnson Mammenga, An embedding theorem, J. Algebra, 325 (2011), 177-185.

[11] J. Coykendall and M. Zafrullah, AP-domains and unique factorization, J. Pure Appl. Algebra, 189 (2004), 27-35.

[12] R. C. Daileda, A non-UFD integral domains in which irreducibles are prime, preprint, http://ramanujan.math.trinity.edu/daileda/teach/m4363s07/nonufd. pdf.

[13] L. Fuchs, Infinite Abelian Groups, Vol. II, Pure and Applied Mathematics, Vol. 36-II, Academic Press, New York-London, 1973.

[14] A. Geroldinger and F. Halter-Koch, Non-Unique Factorizations, Algebraic, Combinatorial and Analytic Theory, Pure and Applied Mathematics, 278, Chapman \& Hall/CRC, Boca Raton, FL, 2006.

[15] A. Geroldinger and W. Hassler, Local tameness of v-noetherian monoids, J. Pure Appl. Algebra, 212(6) (2008), 1509-1524.

[16] A. Geroldinger and W. Hassler, Arithmetic of Mori domains and monoids, J. Algebra, 319(8) (2008), 3419-3463.

[17] A. Geroldinger and A. Reinhart, The monotone catenary degree of monoids of ideals, Internat. J. Algebra Comput., 29(3) (2019), 419-457.

[18] R. Gilmer, Multiplicative Ideal Theory, Pure and Applied Mathematics, No. 12, Marcel Dekker, Inc., New York, 1972. 
[19] R. Gilmer, Commutative Semigroup Rings, Chicago Lectures in Mathematics, University of Chicago Press, Chicago, IL, 1984.

[20] R. Gilmer and T. Parker, Divisibility properties in semigroup rings, Michigan Math. J., 21 (1974), 6586.

[21] R. Gilmer and T. Parker, Semigroup rings as Prüfer rings, Duke Math. J., 41 (1974), 219-230.

[22] R. Gipson and H. Kulosman, Atomic and AP semigroup rings $F[X ; M]$, where $M$ is a submonoid of the additive monoid of nonnegative rational numbers, Int. Electron. J. Algebra, 22 (2017), 133-146.

[23] F. Gotti, On the atomic structure of Puiseux monoids, J. Algebra Appl., 16(7) (2017), 1750126 (20 pp).

[24] F. Gotti, Puiseux monoids and transfer homomorphisms, J. Algebra, 516 (2018), 95-114.

[25] M. Gotti, On the local k-elasticities of Puiseux monoids, Internat. J. Algebra Comput., 29(1) (2019), 147-158.

[26] F. Gotti and C. O'Neill, The elasticity of Puiseux monoids, to appear in J. Commut. Algebra, https://projecteuclid.org/euclid.jca/1523433696; also arXiv 1703.04207v1 [math.AC].

[27] A. Grams, Atomic rings and the ascending chain condition for principal ideals, Proc. Cambridge Philos. Soc., 75 (1974), 321-329.

[28] P. A. Grillet, Commutative Semigroups, Advances in Mathematics (Dordrecht), 2, Kluwer Academic Publishers, Dordrecht, 2001.

[29] F. Halter-Koch, Ideal Systems, An Introduction to Multiplicative Ideal Theory, Monographs and Textbooks in Pure and Applied Mathematics, 211, Marcel Dekker, Inc., New York, 1998.

[30] H. C. Hutchins, Examples of Commutative Rings, Polygonal Publ. House, Washington, N. J., 1981.

[31] P. Jaffard, Les Systems des Ideaux, Dunod, 1960.

[32] I. Kaplansky, Commutative Rings, Revised Edition, The University of Chicago Press, Chicago and London, 1974.

[33] H. Kulosman, A new simple example of an atomic domain which is not ACCP, Adv. Algebra, 12 (2019), 1-7.

[34] R. Matsuda, Torsion-free abelian group rings III, Bull. Fac. Sci., Ibaraki Univ. Ser. A, 9 (1977), 1-49.

[35] D. G. Northcott, Lessons on Rings, Modules and Multiplicities, Cambridge University Press, Cambridge, 1968. 
[36] M. Zafrullah, On a property of pre-Schreier domains, Comm. Algebra, 15(9) (1987), 1895-1920.

Ryan Gipson and Hamid Kulosman (Corresponding Author)

Department of Mathematics

University of Louisville

Louisville, KY 40292, USA

e-mails: ryan.gipson@louisville.edu (R. Gipson)

hamid.kulosman@louisville.edu (H. Kulosman) 